\title{
Suppression of personality variation in boldness during foraging in three-spined sticklebacks
}

\author{
Hannah E. A. MacGregor ${ }^{1}$ (D) $\cdot$ Aislinn Cottage ${ }^{1} \cdot$ Christos C. Ioannou ${ }^{1}$ (D) \\ Received: 2 November 2020 / Revised: 3 March 2021 / Accepted: 9 March 2021 / Published online: 24 March 2021 \\ (C) The Author(s) 2021
}

\begin{abstract}
Consistent inter-individual variation in behaviour within a population, widely referred to as personality variation, can be affected by environmental context. Feedbacks between an individual's behaviour and state can strengthen (positive feedback) or weaken (negative feedback) individual differences when experiences such as predator encounters or winning contests are dependent on behavioural type. We examined the influence of foraging on individual-level consistency in refuge use (a measure of risk-taking, i.e. boldness) in three-spined sticklebacks, Gasterosteus aculeatus, and particularly whether changes in refuge use depended on boldness measured under control conditions. In the control treatment trials with no food, individuals were repeatable in refuge use across repeated trials, and this behavioural consistency did not differ between the start and end of these trials. In contrast, when food was available, individuals showed a higher degree of consistency in refuge use at the start of the trials versus controls but this consistency significantly reduced by the end of the trials. The effect of the opportunity to forage was dependent on behavioural type, with bolder fish varying more in their refuge use between the start and the end of the feeding trials than shyer fish, and boldness positively predicted the likelihood of feeding at the start but not at the end of the trials. This suggests a state-behaviour feedback, but there was no overall trend in how bolder individuals changed their behaviour. Our study shows that personality variation can be suppressed in foraging contexts and a potential but unpredictable role of feedbacks between state and behaviour.
\end{abstract}

\section{Significance statement}

In this experimental study, we examined how foraging influences consistency in risk-taking in individual three-spined sticklebacks. We show that bolder individuals become less consistent in their risk-taking behaviour than shyer individuals during foraging. Some bolder individuals reinforce their risk-taking behaviour, suggesting a positive feedback between state and behaviour, while others converge on the behaviour of shyer individuals, suggesting a negative feedback. In support of a role of satiation in driving negative feedback effects, we found that bolder individuals were more likely to feed at the start but not at the end of the trials. Overall, our findings suggest that foraging can influence personality variation in risk-taking behaviour; however, the role of feedbacks may be unpredictable.

Keywords Animal temperament · risk-taking · repeatability $\cdot$ refuge use $\cdot$ Gasterosteus aculeatus $\cdot$ behavioural type

\section{Introduction}

Individuals of the same species within a population often differ consistently in their behaviour over time and contexts (Magurran et al. 1998; Dall et al. 2004; Sih et al. 2004). For

Communicated by I. Hamilton

Hannah E. A. MacGregor

hannah.macgregor@bristol.ac.uk

1 School of Biological Sciences, University of Bristol, BS8 ITQ, Bristol, UK instance, individuals may be consistent over time in their reaction to environmental stimuli such as food (MacGregor et al. 2020; Szopa-Comley et al. 2020) or predators (Boissy 1995), or express behavioural correlations across contexts, such as being more aggressive to conspecifics and also bolder in the presence of predators (Huntingford 1976). It is increasingly evident that this personality variation can be heritable (van Oers et al. 2004), has fitness consequences (Smith and Blumstein 2008; Moiron et al. 2020), and contributes to a diverse range of ecological and evolutionary processes (Dingemanse and Réale 2005; Dall et al. 2012; Carere and Gherardi 2013). 
Recently, attention has turned to understanding the conditions that promote the expression of animal personality and the interplay between personality and behavioural plasticity (Briffa et al. 2008; Dingemanse et al. 2010; Mathot et al. 2011). On the one hand, plasticity in behavioural traits can allow individuals to respond rapidly and adaptively to changing conditions, including to factors such as predation risk, resources and the social environment (Via et al. 1995; Snell-Rood 2013). On the other hand, personality variation can arise under fluctuating selection where the optimal behavioural phenotype varies over space or time (Mangel 1991; Boon et al. 2007). Conventionally, personality and plasticity in behaviour have been studied independently; however, it is increasingly apparent that they may covary, with individuals differing in their responsiveness to changes in environmental and social conditions (Biro et al. 2010; Westneat et al. 2011; Dingemanse et al. 2012; Laskowski and Bell 2013; Stamps 2016; Bevan et al. 2018).

The effects of environmental and social factors on the expression of behavioural variation may be linked to the state dependence of behaviour, where state variables refer to intrinsic factors (e.g. morphology, physiology, information, fecundity) that influence the balance between the costs and benefits of an animal's behavioural decisions (Houston and McNamara 1999; Dingemanse and Wolf 2010; Sih et al. 2015). It is widely accepted that consistency in behavioural variation may affect, and be affected by, an individual's state. For example, individuals more willing to accept potential risk and leave the safety of a refuge (bolder individuals) can have greater access to food (McDonald et al. 2016) and become satiated, while satiation can reduce the risk-taking behaviour of bold individuals (Nakayama et al. 2012). These effects can result in feedbacks (Sih et al. 2015) that can be positive (the effect of a behaviour on state and the effect of state on behaviour act to reinforce each other) or negative (the effect of behaviour on state and the effect of state on behaviour have opposing effects) (Rands et al. 2003; Luttbeg and Sih 2010).

Positive feedback can reinforce and magnify personality expression while negative feedback can reduce it. In the context of foraging, negative feedback between state and behaviour may occur when individuals with low energy reserves show greater risk-taking behaviour than those with high energy reserves and thus acquire more food allowing them to be more cautious in the future (i.e. the asset protection principle (Clark 1994; Houston and McNamara 1999), although see Rands et al. 2003). However, under high predation risk, positive feedback between state and risk-taking may occur because individuals in good condition are better equipped to escape when confronted with a predator and are therefore willing to take greater risks during foraging (i.e. state-dependent safety, Luttbeg and Sih 2010). In this scenario, individuals in good condition will acquire more resources, reinforcing their condition and helping to maintain personality differences. Despite strong theoretical support, empirical evidence for the effects of state-behaviour feedbacks on animal personality is mixed, and mostly confined to studies demonstrating a correlation between state variables and consistent behavioural traits (Niemelä and Dingemanse 2018). One exception is a recent study of consistent interindividual differences in foraging behaviour and gizzard mass in red knots, where diet quality was found to increase gizzard mass and larger gizzard size was associated with higher food intake, supporting a positive feedback between gizzard mass and foraging behaviour (Mathot et al. 2017).

Fish express consistent inter-individual differences in a range of behaviours related to functionally important tasks, including mating behaviour (Magellan and Magurran 2007), parental care (Budaev et al. 1999), predator avoidance (Kortet et al. 2015) and foraging (MacGregor et al. 2020). Research on the factors that influence the expression of personality in these contexts has mainly focused on the role of predation risk (Brown et al. 2007; Dingemanse et al. 2009; Harris et al. 2010), although increasingly the effects of other environmental variables, including abiotic factors, are being explored (e.g. temperature (Biro et al. 2010), turbidity (Ehlman et al. 2019), salinity (Sommer-Trembo et al. 2017)), as well as the effects of the social environment (McDonald et al. 2016; Bevan et al. 2018). Together, these studies provide evidence that the expression of consistent individual differences in behaviour is highly context-dependent, often varying in response to changes in subtle aspects of the environment and over short time scales.

In natural environments, food resources fluctuate in space and time; prey species must make decisions whether or not to leave the safety of a refuge to forage (Sih 1997). These decisions can have enormous impacts on ecological communities owing to their effects on predation risk, predator-prey dynamics and trophic interactions (Sih et al. 1988; Orrock et al. 2013; Belgrad and Griffen 2016). In three-spined sticklebacks (Gasterosteus aculeatus), refuge use behaviour is known to vary consistently between individuals (Bevan et al. 2018; Szopa-Comley et al. 2020) and is a measure of an individual's willingness to accept potential risk traded off for greater access to resources, also known as boldness (Ioannou et al. 2008; Harcourt et al. 2009; McDonald et al. 2016). In this study, we presented three-spined sticklebacks with either a foraging context (feeding treatment) or a control trial with no food (control treatment) on alternate days for four consecutive days to experimentally test whether the opportunity to forage affected inter-individual consistency in refuge use behaviour between trials at the start compared to the end of trials. We measured consistency within each pair of repeated time segments: the first 5 minutes of the two foraging trials, the final 5 minutes of the foraging trials, the first 5 minutes of the two control trials and the final 5 minutes of the control trials. To examine whether plasticity in refuge use behaviour during the foraging trials varied with boldness, we then tested 
whether changes in refuge use at the start compared to at the end of the feeding treatment trials differed depending on an individuals' mean latency to emerge from the refuge in control trials. Finally, we tested whether the relationship between boldness and the likelihood of feeding varied between the start and the end of the trials.

Despite the likely role of state-behaviour feedbacks in animal personality, evidence for the effects of environmentally induced changes in state on the expression of animal personality is limited. To help address this gap, our study aimed to explicitly test the effect of foraging on the expression of consistent individual differences in refuge use. Actively foraging and consuming food may increase refuge use due to satiation (a negative feedback with boldness) or decrease refuge use as individuals acclimatize more quickly to the area outside the refuge initially perceived as risky (a positive feedback with boldness). If feedback effects are negative due to satiation, we predicted that the opportunity to forage in our feeding treatment would reduce inter-individual consistency in refuge use behaviour at the end compared to the start of the trials. In this scenario, bolder individuals were predicted to increase their refuge use because bolder individuals should consume more food and reduce their risk-taking behaviour more. If feedback effects are positive due to learning and acclimatization, we predicted that the opportunity to forage would reinforce inter-individual consistency in refuge use behaviour because bolder individuals will learn that their environment offers high reward and low risk, increasing their time spent away from the refuge area. We predicted that feedback effects would be strongest in bolder compared to shyer individuals because bolder individuals will interact more with their environment.

\section{Methods}

\section{Study animals}

Three-spined sticklebacks $(37 \pm 7.0 \mathrm{~mm}$, standard body length (SL) $\pm \mathrm{SD}$ at time of testing) were collected from the River Cary, Somerset, UK (ST 469 303) and transported to laboratory facilities. The fish were held for 14 months prior to the experiment in glass tanks $(70 \mathrm{~cm}(\mathrm{~L}) \times 45 \mathrm{~cm}(\mathrm{~W}) \times 37.5 \mathrm{~cm}$ (H)) of approximately 50 individuals each and fed daily with defrosted frozen bloodworm (Chironomid larvae). The tanks were enriched with artificial plants and plastic tubing. The fish were not sexed because the ambient temperature $\left(16^{\circ} \mathrm{C}\right)$ and photocycle (11:13 h light:dark) prevented them from attaining sexual maturation. Sixty-four fish were used in the study.

\section{Experimental set-up}

Experiments took place in a white acrylic plastic arena (136 $(\mathrm{L}) \times 72(\mathrm{~W}) \times 19.5(\mathrm{H}) \mathrm{cm})$ divided into four identical channels $(136(\mathrm{~L}) \times 14.5(\mathrm{~W}) \mathrm{cm})$. Lighting was provided by a florescent lamp positioned at each of the narrow ends of the arena. The arena was sloped lengthways and filled with dechlorinated water $\left(16^{\circ} \mathrm{C}\right)$ varying from 7 to $10 \mathrm{~cm}$ in depth. In the shallow end of each channel was a single refuge consisting of half a terracotta clay plant pot $(10(\mathrm{~L}) \times 11-7$ $(\mathrm{W}) \times 5-3.5(\mathrm{H}) \mathrm{cm}$ ) laid on its side. The exit for each refuge faced towards the shallow end wall of the arena and was $15 \mathrm{~cm}$ from the wall. In the deep end of each channel was a clear petri dish (ø: $9 \mathrm{~cm})$ centred $10 \mathrm{~cm}$ from the wall so that any food within the petri dish could be visible to the fish once they had exited and swum around the refuge. We filmed trials from above with a GoPro Hero5 video camera (resolution: $1920 \times$ 1080, 30 frames per second) positioned centrally $92 \mathrm{~cm}$ above the arena. The camera was connected to an external monitor, allowing observations during trials, and video recording was triggered remotely. The arena was enclosed to camera height with white corrugated plastic to minimize external disturbances.

\section{Experimental protocol}

Experiments were conducted on four batches of sixteen fish over four consecutive weeks (23 October to 16 November 2018). For each fish, testing took place over four consecutive days (Tuesday to Friday). On Monday morning before the first day of experiments, we assigned sixteen fish to four groups of four individuals and transferred them to smaller glass holding tanks $(70(\mathrm{~L}) \times 25(\mathrm{~W}) \times 37.5(\mathrm{H}) \mathrm{cm})$. Assignment was carried out by netting four fish of similar body length from the stock tanks and randomly allocating them to one of the four groups. We repeated this process three more times with different size classes of individuals to create variance in body length within each group that could be used for individual identification. Because the fish were not in reproductive condition, they did not display territorial or aggressive behaviours in the holding tanks. Following assignment, the groups were fed with defrosted frozen bloodworm in the afternoon of the same day. Over the subsequent 4 days, we tested fish once per day with one of two treatment types: feeding or control. All four fish within a group were tested simultaneously, one fish in each of the four channels. Each batch of 16 fish was alternated as to whether they received the feeding or control treatment on the first day of testing and the order of treatments was then alternated between days. The order of testing of the groups was allocated at random each day within the constraint that each group was tested, first, second, third and fourth in their batch over the 4 days. Each individual in the group was allocated to a channel in the arena at random within the constraint that they experienced all four channels over the 4 days.

Trials lasted for 40 minutes. In the feeding treatment, fifty medium-sized ( $\sim 1 \mathrm{~cm}$ long) bloodworms were placed in the petri dish immediately prior to commencing the trial. A large 
number of bloodworm was provided to enable satiation. The presence of food could be detected by the fish based on chemical cues but would not be visible until they had exited and swum around the refuge. To quantify food consumption in the feeding treatment, we subtracted the number of bloodworm remaining at the end of the trial from fifty. At the end of a trial, the fish were immediately transferred to their holding tank. All groups were fed with bloodworm following the last trial in a day to standardize levels of satiation. The arena water was aerated with airstones when not in use. All individuals received four trials except in two cases where two individuals from the same group escaped from their holding tank prior to their second feeding treatment trial. This resulted in a final dataset of 254 trials for 64 individuals.

\section{Video analysis}

Behavioural data were extracted from the video footage using the event recording software BORIS (Friard et al. 2016) by two observers who were allocated trials to process at random and in a random order and who were blind to the identities of individual fish during the data extraction. The channels were subdivided along their long axis into three zones: a refuge area, ending at the closed end of the refuge; a neutral area, beginning at the closed end of the refuge and ending on a tangent with the inner edge of the petri dish; and a feeding area, beginning on a tangent with the inner end of the petri dish and ending at the wall. We quantified the following behaviours from the videos: latency to emerge from the refuge that terminated once the fish had their entire body out of the refuge, which we used to measure boldness (e.g. Brown et al. 2005); the duration of time (to the nearest second) that the fish spent in the refuge area for the start and end 5-minute segments of the trial, where we deemed that a fish had crossed from one zone to another when their head crossed the boundary between zones; and whether a fish fed in each of the start and end 5-minute segments of a trial. If the fish did not emerge during the trial, they were given an emergence latency of $2400 \mathrm{~s}$ to match the length of the trial. One fish was not successfully transferred into the refuge at the start of the second feeding treatment trial and was therefore excluded from analyses of inter-individual consistency in latency to emerge from the refuge.

\section{Statistical analyses}

Statistical analyses were performed in $\mathrm{R}$ version 3.6.0 (R Core Team 2019). The initial analyses tested whether the willingness to accept risk and emerge from the refuge was affected by experimental variables (treatment and trial number) and body length. A negative binomial generalized linear mixed model (GLMM) including treatment, trial number and SL as main effects and individual identity as a random intercept was used to examine the predictors of latency to emerge from the refuge. To test whether individual identity accounted for significant variation in the latency of fish to emerge from the refuge, we then compared the goodness-of-fit (deviance) of the GLMM to the model with individual identity removed using a likelihood ratio test (LRT).

To estimate inter-individual consistency in the latency to emerge from the refuge (with a maximum value of $2400 \mathrm{~s}$ ) in the control and feeding treatments, and the time spent in the refuge area during the start and end 5-minute periods (with a maximum value of $300 \mathrm{~s}$ assigned for each time segment), we used Spearman's rank correlation coefficients due to the statistical issues associated with a large proportion of data points being right-censored (e.g. inflated repeatability, Stamps et al. 2012). To statistically compare the correlation coefficients, we performed randomization tests with 1000 iterations (Manly 1991). For emergence latency, the Spearman's rank correlation coefficient was calculated separately for the control and feeding treatment trials. The difference between these correlations was used as the observed difference in inter-individual consistency in emergence latency between treatments. For each iteration of the randomization, each individual fish's emergence latencies were randomly shuffled between treatments, and the correlation coefficients and their difference were recalculated. We compared the absolute observed difference of the correlation coefficients to the frequency distribution of the absolute randomized expected differences to determine the significance (alpha $=0.05$ ). We used a similar approach to compare the Spearman's rank correlation coefficient in time spent in the refuge area between the start segments of the two feeding trials and, separately, the two end segments. Here, the difference between the start and end correlation coefficients was used as the observed change in consistency between the start and end segments. For each iteration of the randomization, each individual fish's values for the time spent in the refuge area were randomly shuffled between the start and the end segments, and the correlation coefficients and their difference were recalculated. The analysis was repeated for the control treatment trials.

An individual's mean latency to emerge from the refuge in the control treatment trials was used as an estimate of their boldness in further analyses (where smaller values represent bolder fish). To test whether the absolute change or directional change in individuals' refuge use during the feeding trials was associated with boldness, we estimated the Spearman's rank correlation between the mean latency to emerge from the refuge in the control treatment trials and the absolute (i.e. negative values made positive) difference in the time that a fish spent in the refuge area between the start and end of the feeding treatments, using an individual's mean difference in time from the two trials. The correlation test then was repeated using the non-absolute rather than absolute difference. To test for an association between change in refuge use during the 
feeding trials and body length, we also estimated Spearman's rank correlations between SL and the mean absolute and the mean non-absolute differences in time that a fish spent in the refuge area between the start and end of the feeding treatments. GLMMs were not performed due to violation of parametric assumptions.

To test whether there was a change in the relationship between boldness and foraging at the end compared to the start of the feeding trials (as predicted with negative feedback), we used a GLMM with binomial error distribution to test for a significant interaction between mean latency to emerge from the refuge in the control treatment (scaled: mean $=0, \mathrm{SD}=1$ ) and time segment (start of trial or end of trial) on the likelihood that a fish fed. Mean latency to emerge from the refuge in the control treatment, time segment, SL and test order (1st or 2nd) were also included in the model as main effects and individual identity was included as a random effect. The two individuals with data for only one trial were excluded. The likelihood that a fish fed was analysed as a response variable rather than the number of bloodworms consumed per individual because in over half of the trials, no bloodworm was consumed by the focal fish at the start and at the end of the feeding treatment.

Analyses assuming a negative binomial or binomial distribution were checked for model assumptions using diagnostic plots in $\mathrm{R}$ package DHARMa. The statistical significance of fixed effects was tested with likelihood ratio tests in $\mathrm{R}$ package lme4.

\section{Results}

\section{Inter-individual consistency in risk-taking behaviour}

In $13 \%$ of cases $(n=33 / 253)$, the fish did not emerge from the refuge during a trial. The latency to first leave the refuge during a trial was longer for larger fish than for smaller fish (GLMM (negative binomial): $\mathrm{SL}$, estimate $\pm \mathrm{SE}=0.06 \pm$ $\left.0.021, \chi^{2}=7.90, P=0.005\right)$ and increased over the 4 days of trials (trial number: estimate $=0.23 \pm 0.059, \chi^{2}=15.28, P<$ 0.0001 ), but there was no significant effect of treatment (treatment (control as reference level): estimate $=0.07 \pm 0.119, \chi^{2}$ $=0.39, P=0.53)$. There were consistent differences between individuals in their latency to first leave the refuge, including after controlling for body length, treatment and trial number (Individual Identity Intercept: LRT: $\chi^{2}=89.4, P<0.0001$ ).

When analysing the data from the two treatments separately, inter-individual differences in latency to emerge from the refuge were significantly correlated in the control (Spearman's rank correlation: $R_{\mathrm{s}}=0.53, P<0.0001, n=61$ ) and feeding (Spearman's rank correlation: $R_{\mathrm{s}}=0.62, P<$ $0.0001, n=61$ ) treatments. The difference between treatments in the Spearman's rank correlation coefficients in latency to emerge from the refuge did not differ significantly from the frequency distribution of the absolute randomized expected differences, suggesting there was no significant effect of treatment on the consistency in inter-individual differences (observed absolute difference in Spearman's rank correlation = 0.095 , mean expected absolute difference in Spearman's rank correlation $=0.096, P=0.50$, Fig. 1). An individual's mean latency to emerge from the refuge in the control treatment (boldness) was significantly positively correlated with their mean latency to emerge from the refuge in the feeding treatment (Spearman's rank correlation: $R_{\mathrm{s}}=0.75, P<0.0001, n=$ 64).

\section{Effects of opportunity to forage and behavioural type on inter-individual consistency in refuge use}

Inter-individual differences in the time spent in the refuge area were significantly correlated in the start (Spearman's rank correlation: $\left.R_{\mathrm{s}}=0.73, P<0.0001, n=62\right)$ and end $\left(R_{\mathrm{s}}=\right.$ $0.43, P=0.0006, n=62)$ segments of the two feeding treatment trials and almost significantly correlated at the start $\left(R_{\mathrm{s}}=\right.$ $0.24, P=0.06, n=64)$ and significantly correlated at the end $\left(R_{\mathrm{S}}=0.33, P=0.008, n=64\right)$ segments of the two control treatment trials (Fig. 2). In the control treatment, the difference in the Spearman's rank correlation coefficients in the time spent in the refuge area across the start of the trials and across the end of the trials did not differ significantly from the frequency distribution of the absolute randomized expected differences (observed absolute difference in Spearman's rank correlation $=0.092$, mean expected absolute difference in Spearman's rank correlation $=0.132, P=0.57$, Fig. 3a). In contrast, in the feeding treatment, the difference between the

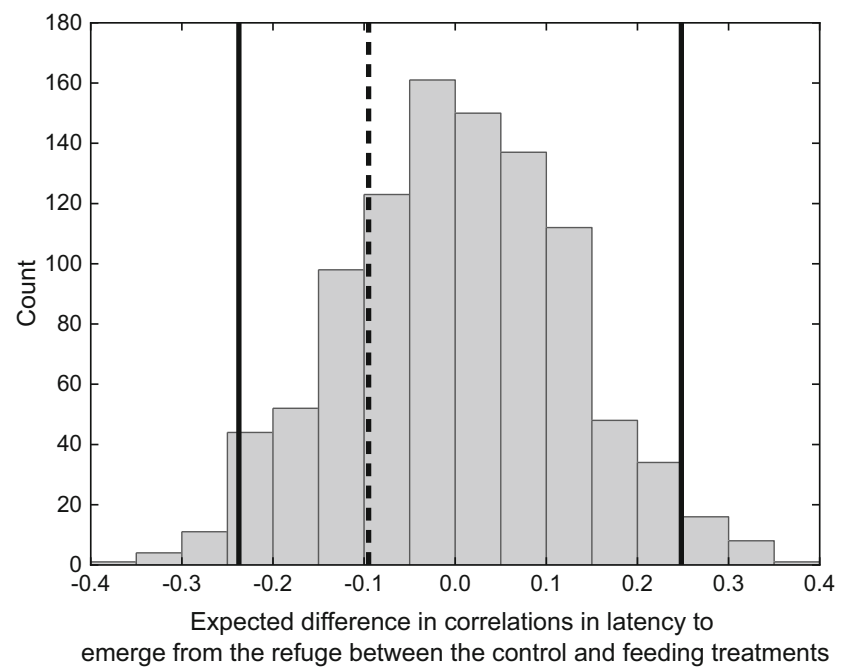

Fig. 1 The expected difference in Spearman's rank correlation coefficients in the latency to emerge from the refuge between the control and feeding treatments. Values are based on 1000 randomizations of the data within individuals. If the observed difference (dashed black line) is outside of the $95 \%$ limits of the randomized data's distribution (solid black lines), it is unlikely that observed difference occurred by chance 
Fig. 2 Correlations between the time spent in the refuge area at the start $(\mathbf{a}, \mathbf{b})$ and at the end $(\mathbf{c}, \mathbf{d})$ of the trials for the control $(\mathbf{a}, \mathbf{c})$ and feeding $(\mathbf{b}, \mathbf{d})$ treatments. Points depict data for individual fish (white, control treatment, and black, feeding treatment)

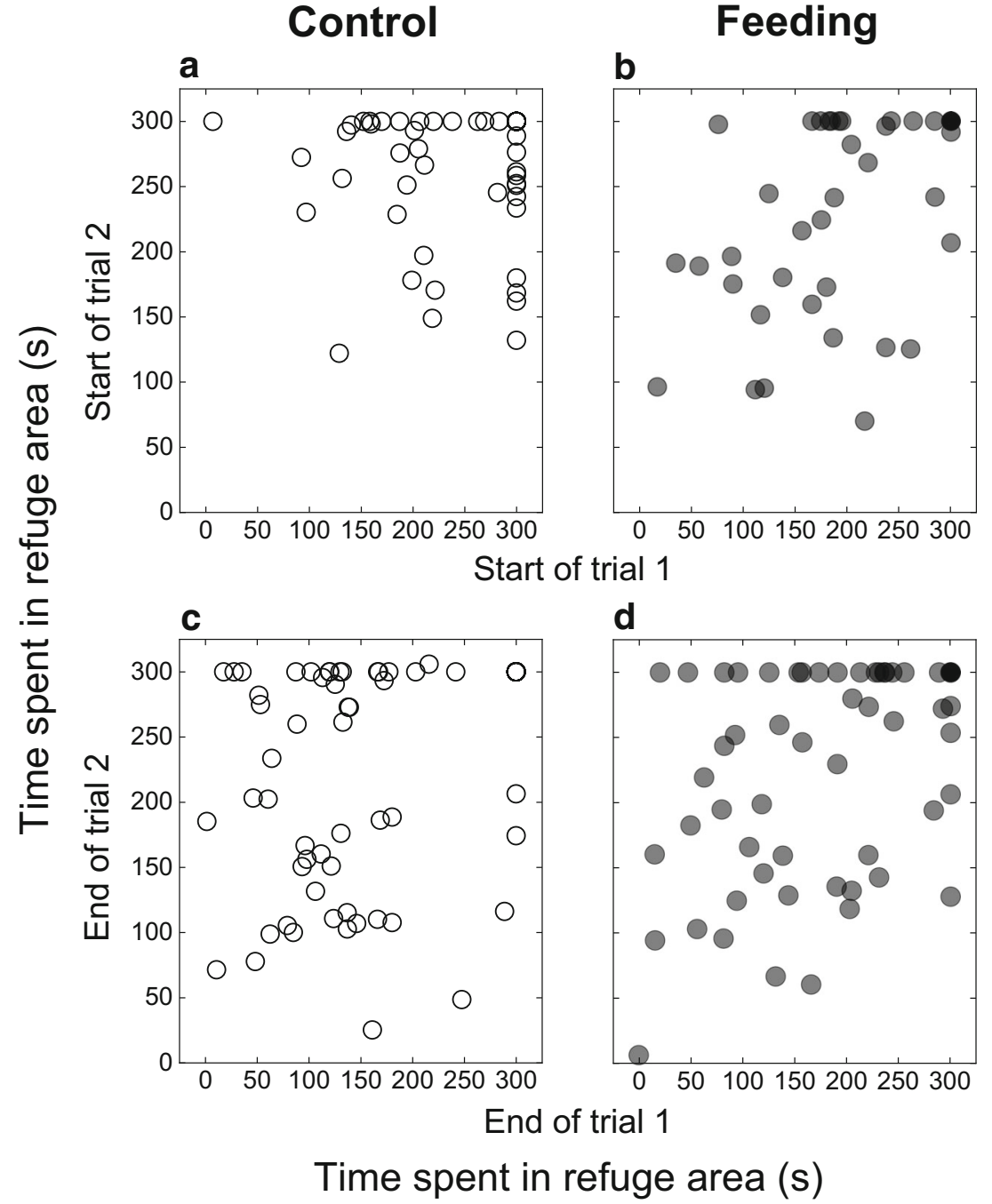

correlation coefficients did differ significantly from expected (observed absolute difference in Spearman's rank correlation $=0.301$, mean expected absolute difference in Spearman's rank correlation $=0.110, P=0.028$, Fig. $3 b$ ). The time in the refuge area was less correlated between the two feeding trials at the end compared to the start of the trials, i.e. the correlation decreased between the start and end segments (Fig. 2b, d).

During the feeding treatment trials, the mean absolute difference in time that fish spent in the refuge area between the start and end of the feeding treatments was significantly negatively correlated with their mean latency to emerge from the refuge in the control treatment (Spearman's rank correlation: $R_{\mathrm{s}}=-0.40, P$ $=0.0012, n=62$, Fig. 4a). In other words, bolder fish changed their refuge use behaviour more between the start and end of the feeding treatment trials than shyer fish. However, there was no significant correlation between the mean latency to emerge from the refuge in the control treatment and the mean non-absolute difference in time spent in the refuge area between the start and end of the feeding treatments (Spearman's rank correlation: $R_{\mathrm{s}}=$
$0.18, P=0.16, n=62$, Fig. $4 \mathrm{~b})$. While shyer fish tended to not change their refuge use at the end compared to the start of the feeding treatment trials, bolder fish showed more variation with some bolder individuals increasing and others decreasing their refuge use at the end compared to the start of the trials (Fig. 4b). Despite there being a significant effect of body length on emergence latency with smaller fish being quicker to emerge, there was no significant correlation between body length and the mean absolute and non-absolute difference in time that a fish spent in the refuge between the start and the end of the feeding treatment trials (mean absolute difference: Spearman's rank correlation: $R_{\mathrm{S}}$ $=0.03, P=0.79, n=62$; mean non-absolute difference: $R_{\mathrm{S}}=$ $0.21, P=0.10, n=62$ ).

\section{Predictors of bloodworm consumption}

Individuals consumed $17 \pm 1.2$ (mean \pm standard error) bloodworms on average in the feeding treatment trials (Fig. 5). In only 4 of 126 trials were all 50 bloodworms consumed, and in 23 trials no bloodworm was consumed. Changes in refuge use 


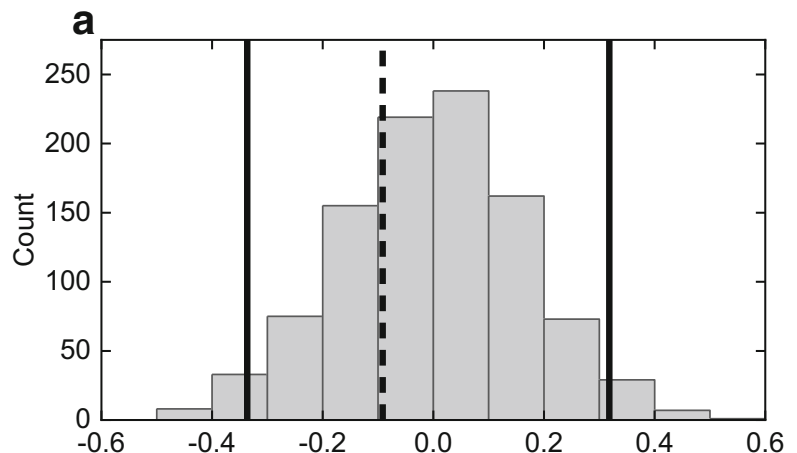

Expected difference in correlations in time spent in the refuge area across the start and across the end of the control treatment

b

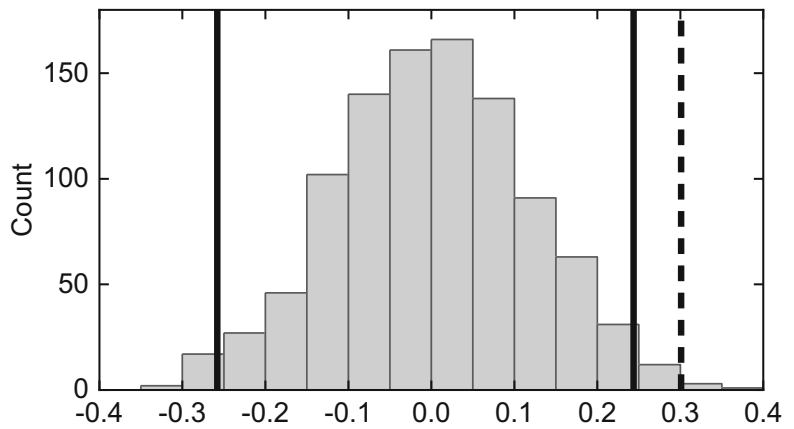

Expected difference in correlations in time spent in the refuge area across the start and across the end of the feeding treatment

Fig. 3 The expected difference in Spearman's rank correlation coefficients in the time spent in the refuge area across the start and across the end of the trials for $\mathbf{a}$ the control treatment and $\mathbf{b}$ the feeding treatment. Values are based on 1000 randomizations of the data within individuals. If the observed difference (dashed black line) is outside of the 95\% limits of the randomized data's distribution (solid black lines), it is unlikely that observed difference occurred by chance

between the start and the end of a trial may be driven by food consumed, either due to positive feedback (e.g. learning about the food reward outside of the refuge) or negative feedback (e.g. from satiation). The likelihood that a fish fed during the feeding trial was predicted by an interaction between boldness and time segment (GLMM (binomial), mean latency to emerge from refuge in control treatment $\times$ time segment: estimate $=1.13 \pm 0.426, \chi^{2}=7.31, P=0.007$, Fig. 6). As expected, bolder fish were more likely to forage at the start of a trial; in contrast, however, indicative of a negative feedback effect of satiation, this was not the case at the end of the trial (Fig. 6). There was also a significant effect of body size on the likelihood of feeding, with smaller fish more likely to feed than larger fish (SL: estimate $=-0.53 \pm 0.185, \chi^{2}=8.76$, $P=0.003)$. Test order was not a significant predictor of the likelihood that a fish fed (test order (1st test as reference level): estimate $\left.=-0.22 \pm 0.330, \chi^{2}=0.43, P=0.51\right)$.

\section{Discussion}

Here, we show that consistent inter-individual differences in refuge use in three-spined sticklebacks were reduced following the opportunity to forage, suggesting that foraging can suppress the expression of personality variation. As in studies of sticklebacks demonstrating that plasticity in behaviour can covary with behavioural type (e.g. Harcourt et al. 2009; Laskowski and Bell 2013; Bevan et al. 2018), we found that bold fish changed their refuge use more than shy fish between the start and end of the feeding treatment trials. This greater change in the behaviour of bolder fish was predicted from state-behaviour feedbacks as bold fish are more likely to interact with the environment. An individual's boldness predicted whether they fed at the start, but not at the end, of the feeding treatment trials, in support of negative feedback between state (satiation) and behaviour (refuge use) causing the observed reduction in personality expression. However, contrary to predictions of negative feedbacks, we did not find
Fig. 4 The relationship between the mean latency to emerge from the refuge in the control treatment (i.e. boldness) and a the mean absolute difference in time that fish spent in the refuge area between the start and end of the feeding treatment and $\mathbf{b}$ the mean non-absolute difference in time that fish spent in the refuge area between the start and end of the feeding treatment
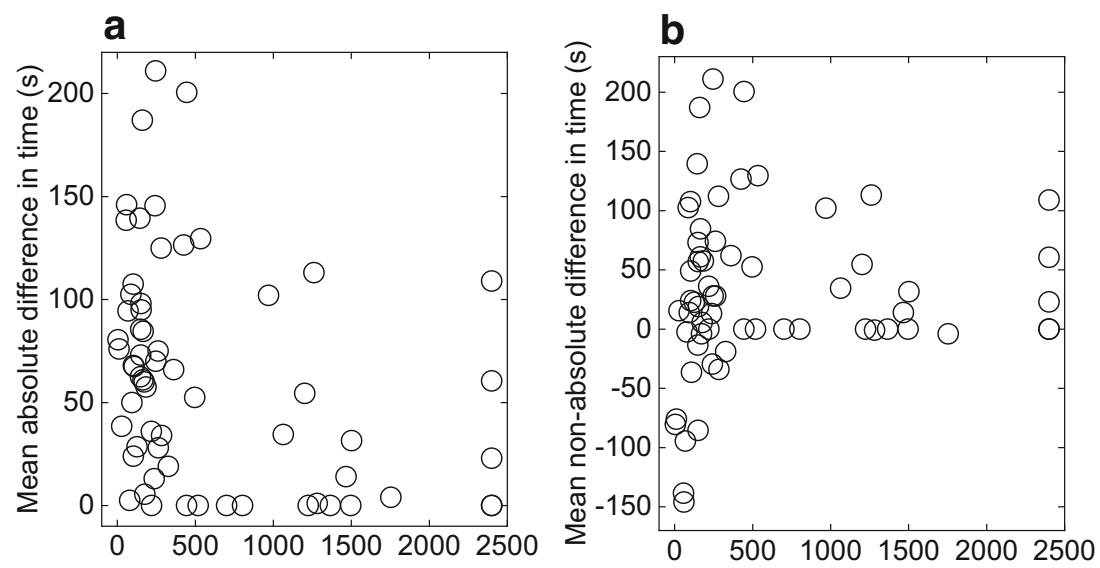

Mean latency to emerge from the refuge in the control treatment (s) 


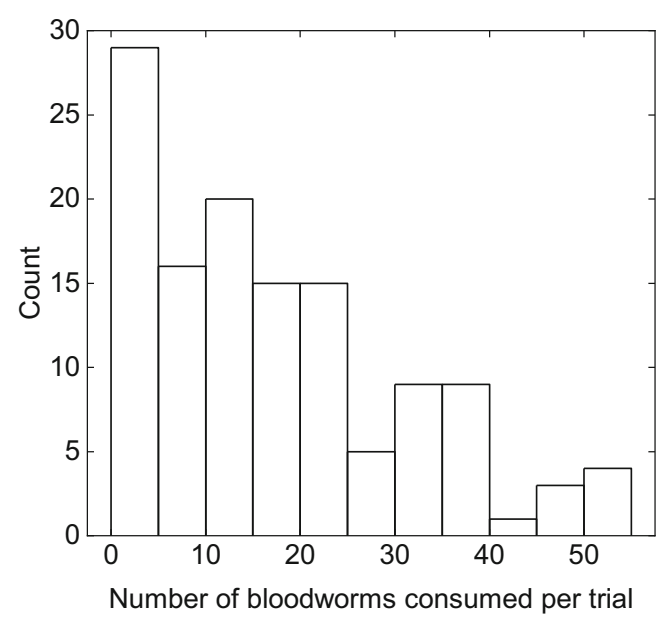

Fig. 5 The number of bloodworms consumed per feeding treatment trial $(n=126)$. Fifty bloodworms were available per trial

evidence that bolder fish used the refuge more at the end compared to the start of the trials, with some bold individuals increasing and others decreasing their refuge use.

While previous studies have often sought to understand how individuals' behaviours change over long time frames, such as during ontogeny (Brommer and Class 2015), very little is known about the processes underlying short-term fluctuations in expression. The state dependency of behaviour is a central concept in theory to explain the origin and maintenance of consistent behavioural variation but could also provide a mechanistic explanation for breakdowns in personality expression

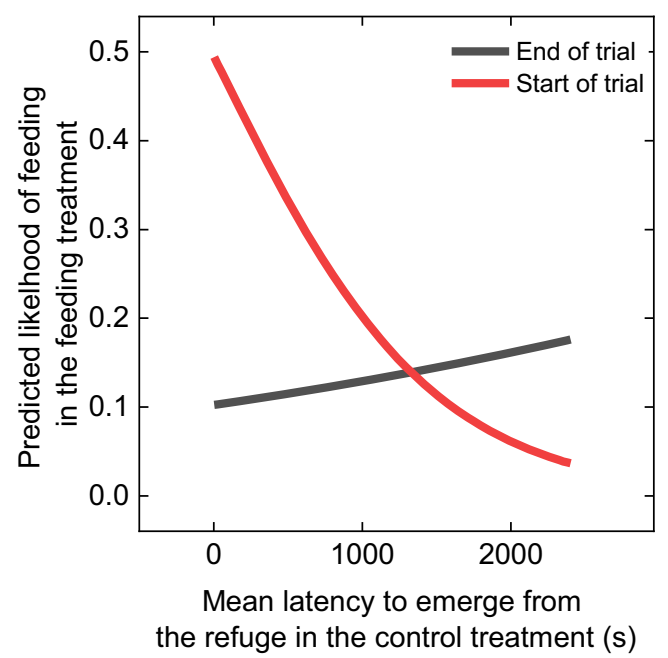

Fig. 6 The predicted interaction between an individual's mean latency to emerge from the refuge in the control treatment and time segment (start of trial or end of trial) on the likelihood that a fish fed in the feeding treatment. Predictions are generated from a GLMM (binomial) including mean latency to emerge from the refuge in the control treatment, time segment, test order and SL as main effects. The intermediate effect of test order and mean SL and were used in the generation of predictions when state variables are labile and produce a negative feedback on behaviour (Dingemanse and Wolf 2010; Sih et al. 2015). In support of this, reduced inter-individual consistency in refuge use during the feeding trials was associated with the opportunity for individuals to increase their nutritional state, and no change in inter-individual consistency was observed in control trials without food. Furthermore, the absence of a significant difference in the first time to leave the refuge (i.e. the emergence latency) between the treatments suggested that it was encountering the food directly rather than the presence of food olfactory cues that affected the inter-individual consistency in behaviour. This also suggests that feedback effects between encountering the food and reduced inter-individual consistency in refuge use were short-lived in our experiment and did not carry over between days.

Previous studies on fish have shown that bolder individuals are more likely to feed in risky contexts (McDonald et al. 2016) but are also more at risk of predation (Bell and Sih 2007; Balaban-Feld et al. 2019), and that nutritional state can influence foraging behaviour (Salvanes and Hart 1998). Therefore, we predicted that a satiation effect in the feeding treatment trials would be strongest in bolder individuals, and, as a result, bolder individuals would change their behaviour more than shy individuals and in the direction of increased refuge use at the end compared to the start of the trials (due to being satiated and the potential risks associated with being away from the refuge). However, counter to expectation, our results did not support directionality to the behavioural changes in bolder individuals, with some bolder individuals expressing even bolder behaviour (more time away from the refuge) and others converging on the refuge use behaviour of shy fish (as predicted due to negative feedback effects between satiation and refuge use). Some of this variation among bold fish could be explained by variation in body size, because smaller fish should satiate more quickly (Brett 1971; Ende et al. 2018), although we did not find a strong correlation between body size and change in refuge use during the feeding treatment trials. An alternative explanation is that the motivation to feed away from a refuge over repeated foraging trips close together in time initially increases with acclimatization and learning about the resource and then reduces with satiation. Such a trend over time was documented by McDonald et al. (McDonald et al. 2016) in shoals of three-spined sticklebacks. In our experiment, some bold fish may have been in the first phase, reducing their refuge use at the end of the trials, while others were in the satiation phase, increasing their refuge use at the end of the trials. In support of a role of negative feedback between satiation and personality, we found a significant interaction between time segment and boldness, with boldness positively predicting the likelihood of feeding at the start but not at the end of the trials.

Inconsistency in behaviour during foraging could have an important adaptive function, for example, by making 
individuals less predictable to predators or competitors if encountering the same individuals repeatably (Briffa 2013; Chang et al. 2017), weakening the strength of directional selection on boldness behaviour and its correlated traits. More generally, inconsistency in the expression of phenotypes may have important evolutionary consequences by potentially weakening evolutionary responses to changes in the environment. Future work that considers the context-dependent expression of animal personality will help to better understand the selection pressures that shape consistent inter-individual differences in behaviour. There is growing evidence of how boldness behaviour such as refuge use can have ecological consequences, including influences on population dynamics via individuals' growth, survival and reproductive success, and on trophic interactions via the effects on the costs and benefits of different predator strategies (Sih et al. 1988; Orrock et al. 2013; Belgrad and Griffen 2016). However, one key outstanding question is in the ecological implications of covariation between personality and behavioural plasticity. Our study suggests this could be particularly challenging to address when behavioural plasticity is unpredictable due to unpredictable state-behaviour feedbacks.

Supplementary Information The online version contains supplementary material available at https://doi.org/10.1007/s00265-021-03007-2.

Acknowledgements We thank Andrew Szopa-Comley and two anonymous reviewers for their constructive comments on the manuscript. We are very grateful to Sarah Washington for assistance with carrying out the experiment. This work was supported by the Natural Environment Research Council grant number NE/P012639/1 awarded to CCI.

Code availability The code used to conduct the analyses is available on request.

Authors' contributions HEAM and CCI designed and coordinated the study. AC conducted the experiment and collected behavioural data. HEAM analysed the data and HEAM and CCI wrote the manuscript. All authors read and approved the final version.

Funding This work was supported by the Natural Environment Research Council grant number NE/P012639/1 awarded to CCI.

Data availability The data analysed in this study are available as electronic supplementary material.

\section{Declarations}

Ethics approval All procedures regarding the use of animals in research followed the United Kingdom guidelines and were approved by the institutional ethics committee (UIN UB/17/060).

Consent to participate Not applicable.

Consent for publication Not applicable.

Conflicts of interest The authors declare no competing interests.
Open Access This article is licensed under a Creative Commons Attribution 4.0 International License, which permits use, sharing, adaptation, distribution and reproduction in any medium or format, as long as you give appropriate credit to the original author(s) and the source, provide a link to the Creative Commons licence, and indicate if changes were made. The images or other third party material in this article are included in the article's Creative Commons licence, unless indicated otherwise in a credit line to the material. If material is not included in the article's Creative Commons licence and your intended use is not permitted by statutory regulation or exceeds the permitted use, you will need to obtain permission directly from the copyright holder. To view a copy of this licence, visit http://creativecommons.org/licenses/by/4.0/.

\section{References}

Balaban-Feld J, Mitchell WA, Kotler BP, Vijayan S, Elem LTT, Rosenzweig ML, Abramsky Z (2019) Individual willingness to leave a safe refuge and the trade-off between food and safety: a test with social fish. Proc R Soc B 286:20190826

Belgrad BA, Griffen BD (2016) Predator and prey interactions mediated by prey personality and predator hunting mode. Proc R Soc B 283: 20160408

Bell AM, Sih A (2007) Exposure to predation generates personality in threespined sticklebacks (Gasterosteus aculeatus). Ecol Lett 10:828-834

Bevan PA, Gosetto I, Jenkins ER, Barnes I, Ioannou CC (2018) Regulation between personality traits: individual social tendencies modulate whether boldness and leadership are correlated. Proc R Soc B 285:20180829

Biro PA, Beckmann C, Stamps JA (2010) Small within-day increases in temperature affects boldness and alters personality in coral reef fish. Proc R Soc Lond B 277:71-77

Boissy A (1995) Fear and fearfulness in animals. Q Rev Biol 70:165-191

Boon AK, Réale D, Boutin S (2007) The interaction between personality, offspring fitness and food abundance in North American red squirrels. Ecol Lett 10:1094-1104

Brett JR (1971) Satiation time, appetite, and maximum food intake of sockeye salmon (Oncorhynchus nerka). J Fish Res Board Can 28:409-415

Briffa M (2013) Plastic proteans: reduced predictability in the face of predation risk in hermit crabs. Biol Lett 9:20130592

Briffa M, Rundle SD, Fryer A (2008) Comparing the strength of behavioural plasticity and consistency across situations: animal personalities in the hermit crab Pagurus bernhardus. Proc R Soc Lond B 275:1305-1311

Brommer JE, Class B (2015) The importance of genotype-by-age interactions for the development of repeatable behaviour and correlated behaviours over lifetime. Front Zool 12:S2

Brown C, Jones F, Braithwaite V (2005) In situ examination of boldnessshyness traits in the tropical poeciliid, Brachyraphis episcopi. Anim Behav 70:1003-1009

Brown C, Burgess F, Braithwaite VA (2007) Heritable and experiential effects on boldness in a tropical poeciliid. Behav Ecol Sociobiol 62:237-243

Budaev SV, Zworykin DD, Mochek AD (1999) Individual differences in parental care and behaviour profile in the convict cichlid: a correlation study. Anim Behav 58:195-202

Carere C, Gherardi F (2013) Animal personalities matter for biological invasions. Trends Ecol Evol 28:5-6

Chang C, Teo HY, Norma-Rashid Y, Li D (2017) Predator personality and prey behavioural predictability jointly determine foraging performance. Sci Rep 7:40734

Clark CW (1994) Antipredator behaviour and the asset-protection principle. Behav Ecol 5:159-170

Dall SRX, Houston AI, McNamara JM (2004) The behavioural ecology of personality: consistent individual differences from an adaptive perspective. Ecol Lett 7:734-739 
Dall SRX, Bell AM, Bolnick DI, Ratnieks FLW (2012) An evolutionary ecology of individual differences. Ecol Lett 15:1189-1198

Dingemanse NJ, Réale D (2005) Natural selection and animal personality. Behaviour 142:1159-1184

Dingemanse NJ, Wolf M (2010) Recent models for adaptive personality differences: a review. Phil Trans R Soc B 365:3947-3958

Dingemanse NJ, van der Plas F, Wright J, Reale D, Schrama M, Roff DA, van der Zee E, Barber I (2009) Individual experience and evolutionary history of predation affect expression of heritable variation in fish personality and morphology. Proc R Soc Lond B 276:1285-1293

Dingemanse NJ, Kazem AJ, Reale D, Wright J (2010) Behavioural reaction norms: animal personality meets individual plasticity. Trends Ecol Evol 25:81-89

Dingemanse NJ, Bouwman KM, van de Pol M, van Overveld T, Patrick SC, Matthysen E, Quinn JL (2012) Variation in personality and behavioural plasticity across four populations of the great tit Parus major. J Anim Ecol 81:116-126

Ehlman SM, Halpin R, Jones C, Munson A, Pollack L, Sih A (2019) Intermediate turbidity elicits the greatest antipredator response and generates repeatable behaviour in mosquitofish. Anim Behav 158:101-108

Ende SSW, Thiele R, Schrama JW, Verreth JAJ (2018) The influence of prey density and fish size on prey consumption in common sole (Solea solea L.). Aquat Living Resour 31:16

Friard O, Gamba M, Fitzjohn R (2016) BORIS: a free, versatile opensource event-logging software for video/audio coding and live observations. Methods Ecol Evol 7:1325-1330

Harcourt JL, Ang TZ, Sweetman G, Johnstone RA, Manica A (2009) Social feedback and the emergence of leaders and followers. Curr Biol 19:248-252

Harris S, Ramnarine IW, Smith HG, Pettersson LB (2010) Picking personalities apart: estimating the influence of predation, sex and body size on boldness in the guppy Poecilia reticulata. Oikos 119:1711-1718

Houston AI, McNamara JM (1999) Models of adaptive behaviour: an approach based on state. Cambridge University Press, Cambridge

Huntingford FA (1976) The relationship between anti-predator behaviour and aggression among conspecifics in the three-spined stickleback, Gasterosteus aculeatus. Anim Behav 24:245-260

Ioannou CC, Payne M, Krause J (2008) Ecological consequences of the bold-shy continuum: the effect of predator boldness on prey risk. Oecologia 157:177-182

Kortet R, Sirkka I, Lai Y-T, Vainikka A, Kekäläinen J (2015) Personality differences in two minnow populations that differ in their parasitism and predation risk. Front Ecol Evol 3:9

Laskowski KL, Bell AM (2013) Competition avoidance drives individual differences in response to a changing food resource in sticklebacks. Ecol Lett 16:746-753

Luttbeg B, Sih A (2010) Risk, resources and state-dependent adaptive behavioural syndromes. Phil Trans R Soc B 365:3977-3990

MacGregor HEA, Herbert-Read JE, Ioannou CC (2020) Information can explain the dynamics of group order in animal collective behaviour. Nat Commun 11:2737

Magellan K, Magurran AE (2007) Behavioural profiles: individual consistency in male mating behaviour under varying sex ratios. Anim Behav 74:1545-1550

Magurran AE, May RM, Wilson DS (1998) Adaptive individual differences within single populations. Phil Trans R Soc B 353:199-205

Mangel M (1991) Adaptive walks on behavioural landscapes and the evolution of optimal behaviour by natural selection. Evol Ecol 5:30-39

Manly BFJ (1991) Randomization and Monte Carlo methods in biology. Chapman and Hall, London

Mathot KJ, van den Hout PJ, Piersma T, Kempenaers B, Réale D, Dingemanse NJ (2011) Disentangling the roles of frequency-vs. state-dependence in generating individual differences in behavioural plasticity. Ecol Lett 14:1254-1262
Mathot KJ, Dekinga A, Piersma T, Sandercock B (2017) An experimental test of state-behaviour feedbacks: gizzard mass and foraging behaviour in red knots. Funct Ecol 31:1111-1121

McDonald ND, Rands SA, Hill F, Elder C, Ioannou CC (2016) Consensus and experience trump leadership, suppressing individual personality during social foraging. Sci Adv 2:e1600892

Moiron M, Laskowski KL, Niemelä PT (2020) Individual differences in behaviour explain variation in survival: a meta-analysis. Ecol Lett 23:399-408

Nakayama S, Johnstone RA, Manica A (2012) Temperament and hunger interact to determine the emergence of leaders in pairs of foraging fish. PLoS ONE 7:e43747

Niemelä PT, Dingemanse NJ (2018) Meta-analysis reveals weak associations between intrinsic state and personality. Proc R Soc B 285:20172823

Orrock JL, Preisser EL, Grabowski JH, Trussell GC (2013) The cost of safety: Refuges increase the impact of predation risk in aquatic systems. Ecology 94:573-579

Rands AS, Cowlishaw G, Pettifor AR, Rowcliffe JM, Johnstone AR (2003) Spontaneous emergence of leaders and followers in foraging pairs. Nature 423:432-434

R Core Team (2019), R: A language and environment for statistical computing. R Foundation for Statistical Computing, Vienna, Austria. https://www.R-project.org/

Salvanes AGV, Hart PJB (1998) Individual variability in state-dependent feeding behaviour in three-spined sticklebacks. Anim Behav 55:1349-1359

Sih A (1997) To hide or not to hide? Refuge use in a fluctuating environment. Trends Ecol Evol 12:375-376

Sih A, Petranka JW, Kats LB (1988) The dynamics of prey refuge use: A model and tests with sunfish and salamander larvae. Am Nat 132:463483

Sih A, Bell A, Johnson JC (2004) Behavioural syndromes: an ecological and evolutionary overview. Trends Ecol Evol 19:372-378

Sih A, Mathot KJ, Moiron M, Montiglio PO, Wolf M, Dingemanse NJ (2015) Animal personality and state-behaviour feedbacks: a review and guide for empiricists. Trends Ecol Evol 30:50-60

Smith BR, Blumstein DT (2008) Fitness consequences of personality: a meta-analysis. Behav Ecol 19:448-455

Snell-Rood EC (2013) An overview of the evolutionary causes and consequences of behavioural plasticity. Anim Behav 85:1004-1011

Sommer-Trembo C, Petry AC, Gomes Silva G, Vurusic SM, Gismann J, Baier J, Krause S, Iorio JAC, Riesch R, Plath M (2017) Predation risk and abiotic habitat parameters affect personality traits in extremophile populations of a neotropical fish (Poecilia vivipara). Ecol Evol 7:6570-6581

Stamps JA (2016) Individual differences in behavioural plasticities. Biol Rev 91:534-567

Stamps JA, Briffa M, Biro PA (2012) Unpredictable animals: individual differences in intraindividual variability (IIV). Anim Behav 83: $1325-1334$

Szopa-Comley AW, Donald WG, Ioannou CC (2020) Predator personality and prey detection: inter-individual variation in responses to cryptic and conspicuous prey. Behav Ecol Sociobiol 74:70

van Oers K, Drent PJ, de Goede P, van Noordwijk AJ (2004) Realized heritability and repeatability of risk-taking behaviour in relation to avian personalities. Proc R Soc Lond B 271:65-73

Via S, Gomulkiewicz R, de Jong G, Scheiner SM, Schlichting CD, van Tienderen PH (1995) Adaptive phenotypic plasticity: consensus and controversy. Trends Ecol Evol 10:212-217

Westneat DF, Hatch MI, Wetzel DP, Ensminger AL (2011) Individual variation in parental care reaction norms: integration of personality and plasticity. Am Nat 178:652-667

Publisher's Note Springer Nature remains neutral with regard to jurisdictional claims in published maps and institutional affiliations. 